\title{
FISIONOMIA E ESTRUTURA DE VEGETAÇÃO DE CAATINGA EM DIFERENTES AMBIENTES EM SERRA TALHADA - PERNAMBUCO
}

\author{
PHYSIOGNOMY AND VEGETATION STRUCTURE IN DIFFERENT ENVIRONMENTS OF \\ "CAATINGA” IN “SERRA TALHADA” PERNAMBUCO STATE, BRAZIL
}

\author{
Séfora Gil Gomes de Farias ${ }^{1}$ Maria Jesus Nogueira Rodal ${ }^{2}$ André Laurênio de Melo ${ }^{3}$ \\ Maria Amanda Menezes Silva ${ }^{4}$ André Luiz Alves de Lima ${ }^{5}$
}

\begin{abstract}
RESUMO
Este estudo foi realizado com o objetivo de investigar a influência da heterogeneidade espacial dos fatores abióticos sobre os padrões espaciais da estrutura da vegetação do componente lenhoso e sua regeneração em uma área de caatinga, no sertão de Pernambuco. O estudo foi realizado em uma área de caatinga localizada no Parque Estadual da Mata da Pimenteira, município de Serra Talhada, Pernambuco. A amostragem foi dividida em dois ambientes, um próximo ao curso d'água com vegetação mais densa (I) e outro a $100 \mathrm{~m}$ do curso d'água com vegetação mais aberta(II). Em cada ambiente foram instaladas quatro parcelas permanentes de $20 \times 50 \mathrm{~m}$ subdivididas em 10 parcelas de $10 \times 10 \mathrm{~m}$, nas quais foram medidos os indivíduos vivos com diâmetro do caule ao nível do solo (DNS) $\geq 3 \mathrm{~cm}$ e altura total $\geq 1 \mathrm{~m}$. Em um dos vértices de cada parcela foi plotada uma subparcela de $2 \times 2 \mathrm{~m}$ para medir a altura e o diâmetro dos indivíduos das espécies amostradas no componente lenhoso com DNS entre 0,5 e $2,9 \mathrm{~cm}$ (regeneração). Foram calculados os descritores fitossociológicos gerais da comunidade dos diferentes ambientes e avaliada a distribuição de indivíduos em histogramas, tanto do componente lenhoso como para a regeneração. Após verificação da normalidade pelo teste Kolmogorov-Smirnov, os valores dos descritores fitossociológicos por parcela foram comparados entre os ambientes. Nos dados com distribuição normal, foi empregada a análise de variância (ANOVA) de um critério. Dados sem distribuição normal foram analisados pelo teste não paramétrico de MannWhitney. Os ambientes apresentaram diferenças significativas quanto ao número de indivíduos $(4047,5$ versus 3332,5 ind.ha $\left.^{-1}\right)$ e área basal $\left(23,6\right.$ versus $17,6 \mathrm{~m}^{2}$. ha- $\left.{ }^{-1}\right)$, com valores significativamente superiores no ambiente I, padrão oposto ao da regeneração em que o número de indivíduos e a área basal foram maiores no ambiente II (9187,5 versus 10937,5 ind.ha $\left.{ }^{-1}\right)$ e (1,9 versus $2,5 \mathrm{~m}^{2}$. ha $\left.\mathrm{a}^{-1}\right)$, respectivamente. No total foram amostradas 50 espécies (incluindo o componente arbóreo e regenerante), havendo diferenças significativas em termos de diversidade entre os ambientes nos diferentes estratos, embora a similaridade seja superior a $80 \%$. A maioria das variáveis químicas e texturais dos solos não diferiram estatisticamente, com exceção dos teores de $\mathrm{Al}^{3+}$ que apresentou teores superiores no ambiente I. Das demais variáveis edáficas, apenas a pedregosidade diferiu entre os ambientes I e II (3,87 versus $1,47 \%)$. A distinção fisionômica da vegetação entre os ambientes é resultado da presença de populações com indivíduos de maiores alturas e diâmetros no ambiente I. A heterogeneidade espacial das variáveis ambientais encontrada nos ambientes estudados,
\end{abstract}

1 Engenheira Florestal, Dr ${ }^{\text {a }}$, Professora da Universidade Federal do Piauí, Campus Professora Cinobelina Elvas, BR 135, km 3, Bairro Planalto Horizonte, CEP 64900-000, Bom Jesus (PI), Brasil. seflora@gmail.com

2 Bióloga, Dra ., Professora do Departamento de Biologia, Laboratório de Fitossociologia, Universidade Federal Rural de Pernambuco, Rua D. Manoel de Medeiros, s/n, Dois Irmãos, CEP 52.171-900, Recife (PE), Brasil. mrodal@terra.com.br

3 Biólogo, Dr., Professor da Unidade Acadêmica de Serra Talhada, Universidade Federal Rural de Pernambuco, Fazenda Saco, s/n, Caixa Postal 063, CEP 56900-000, Serra Talhada (PE), Brasil. andrelaurenio@yahoo.com.br.

4 Bióloga, Dra., Professora do Departamento de Engenharia Ambiental, Instituto Federal do Ceará, Av. José de Freitas Queiroz, 5000, Bairro Cedro, CEP 63902-580, Quixadá (CE), Brasil. amandamenezesmsn@hotmail.com

5 Biólogo, Dr., Professor da Unidade Acadêmica de Serra Talhada, Universidade Federal Rural de Pernambuco, Fazenda Saco, s/n, Caixa Postal 063, CEP 56900-000, Serra Talhada (PE), Brasil. andrelimabotanica@yahoo.com.br

Recebido para publicação em 30/04/2012 e aceito em 3/09/2014 
explicou parte das variações das características florístico-estrutural da vegetação.

Palavras-chave: semiárido; sazonalidade; fatores ambientais; heterogeneidade espacial.

\begin{abstract}
This study aimed to investigate the influence of spatial heterogeneity of abiotic factors on the spatial patterns of vegetation structure of the woody component and of its regeneration; it was carried out in an area of "caatinga" located in the Parque Estadual Mata of Pimenteira, municipality of "Serra Talhada", interior of Pernambuco State, Brazil. The sample was divided into two environments, one near the watercourse with denser vegetation (I) and another at 100 meters away from the watercourse with more open vegetation (II). In each environment, four permanent plots of $20 \times 50 \mathrm{~m}$ were installed and divided into ten plots of $10 \times 10 \mathrm{~m}$; alive individuals with stem ground level diameter (GD) of $\geq 3 \mathrm{~cm}$ and total height of $\geq 1 \mathrm{~m}$ were measured. In one corner of each plot, a sub-plot of $2 \times 2 \mathrm{~m}$ was allocated to measure the individuals' height and diameter of the sampled species in woody component with GD from 0.5 to $2.9 \mathrm{~cm}$ (regeneration). Community's general phytosociological descriptors of the different environments were calculated and the individuals' distribution in histograms was evaluated for the woody component as for regeneration. After testing for normality using Kolmogorov-Smirnov test, values of phytosociological descriptors per plot were compared between the environments. Analysis of variance (ANOVA) of a criterion was used for data with normal distribution. Data without normal distribution were analyzed by non-parametric MannWhitney test. Environments showed significant differences in the number of individuals (4047.5 versus 3332.5 ind.ha $\mathrm{a}^{-1}$ ) and basal area (23.6 versus $\left.17.6 \mathrm{~m}^{2} . \mathrm{ha}^{-1}\right)$ with significantly higher values in environment I; this pattern was opposite to the regenerating pattern in which the number of individuals and basal area were higher in the environment II (9187.5 vs. 10937.5 ind.ha $^{-1}$ and $1.9 v s .2 .5 \mathrm{~m}^{2} . \mathrm{ha}^{-1}$, respectively). In total, fifty species, including woody and regenerating component, were sampled; significant differences in diversity were found between environments in the different strata, although the similarity was more than $80 \%$. Most chemical and textural soil variables did not differ statistically, except for levels of $\mathrm{Al}^{3+}$, which showed higher levels in the environment I. Among other edaphic variables, only the stony variables differed between environments I and II (3.87 versus $1.47 \%$ ). The vegetation physiognomic distinction between the environments is result from populations' presence of individuals with greater heights and diameters in the environment $I$. The spatial heterogeneity of environmental variables found in the studied environments explained part of changes in floristic and structural characteristics of the vegetation.
\end{abstract}

Keywords: semiarid; seasonality; environmental factors; spatial heterogeneity.

\section{INTRODUÇÃO}

As áreas tropicais sazonalmente secas representam aproximadamente $42 \%$ da vegetação tropical e subtropical do globo terrestre (MURPHY; LUGO, 1995), onde se encontra o semiárido do Nordeste do Brasil, no qual predomina uma vegetação conhecida como caatinga. Trata-se de um dos tipos vegetacionais brasileiros menos conhecidos e mais negligenciados quanto à conservação de sua biodiversidade. Essa formação apresenta uma grande variabilidade fisionômica, como resultado da elevada variação interna nas condições ambientais, principalmente climáticas e pedológicas (RODAL; SAMPAIO, 2002).

Nesse sentido, Andrade-Lima (1981) apontou a heterogeneidade de fatores climáticos, geomorfológicos e edáficos como os principais fatores geradores da grande variabilidade florística e fisionômica encontrada no semiárido nordestino. Para este autor, a caatinga sensu stricto é a tipologia vegetacional de maior extensão e ocorre basicamente na chamada depressão sertaneja, a qual representa um conjunto de pediplanos rodeado ou entremeado, segundo Rodal e Sampaio (2002), por relevos como: chapadas, bacias sedimentares e serras e maciços com variadas extensões, muitos deles não mapeáveis em escalas inferiores a 1:2.000.000.

Dentre as diversas tentativas de identificação dos diferentes tipos de caatinga sensu stricto, as caracterizações apresentadas por autores como Andrade-Lima (1981) e Veloso et al. (1991) apontam na direção de que, em uma mesma região com mesmo tipo de solo, as diferenças fisionômicas 
encontradas estão relacionadas com as variações ambientais como a proximidade de serras e corpos de água e as alterações de relevo, relacionadas ao escoamento superficial. Autores como Amorim, Sampaio e Araujo (2005) e Rodal, Costa e Linse-Silva (2008a) relataram que as alterações fisionômicas em escala local da caatinga sensu stricto são facilmente perceptíveis e na maioria das vezes, conforme mencionado acima, estão associadas a mudanças nos fatores abióticos. Além disso, observaram que as plantas em áreas de vales apresentaram maior porte quando comparadas às de áreas de lajedos e solos rasos, associando essas diferenças à maior ou menor disponibilidade hídrica encontrada nessas áreas.

Os dados quantitativos fornecidos por Rodal, Costa e Lins-e-Silva (2008a), sobre a vegetação lenhosa em termos de distribuição de diâmetros e alturas, em dois habitat típicos caatinga: do pediplano da depressão e das áreas próximas a riachos e serras, mostraram que a separação fisionômica entre os dois habitat é resultado da presença de indivíduos de maiores diâmetros e alturas. Contudo, o foco de estudos florísticos e fitossociológicos nesses ambientes tem sido centrado no componente arbóreo, no qual está concentrada a maior parte da biomassa de maior valor de mercado. O conhecimento sobre a regeneração natural dessa vegetação ainda é bastante incipiente. Estudos em uma escala menor, envolvendo uma análise mais minuciosa do componente arbóreo e de sua regeneração e sua relação com as características do local, são praticamente inexistentes em áreas de caatinga. Estudos dessa natureza contribuirão para suprir as lacunas nos estudos ecológicos dessa vegetação, facilitando a compreensão das complexas interações entre vegetação e ambiente.

Tais resultados registrados em áreas de caatinga remetem à perspectiva de que os fatores abióticos servem como filtros ambientais (BEGON; TOWNSEND; HARPER, 2007). Na perspectiva de que diferenças entre a disponibilidade de recursos seja um fator que modula a estrutura de assembleias de plantas, pode-se supor que, ambientes com maior disponibilidade de recursos apresentam assembleias com estruturas mais complexas e que diferenças nos habitat como características pedológicas e proximidade de serras e de riachos, dentro de uma mesma unidade ambiental, no caso trechos de caatinga sensu stricto, possam explicar em parte a heterogeneidade fisionômica. Deste modo, este trabalho pretende responder as seguintes questões: 1) A vegetação de duas áreas de caatinga contíguas e com as mesmas características de solo situadas em diferentes distâncias de um curso d'água apresentam fisionomias diferentes?; 2) As variáveis ambientais influenciam a distribuição das espécies e a estrutura de diâmetro e altura das populações amostradas nos dois ambientes? e 3) A estrutura e a fisionomia da regeneração segue os mesmos padrões do componente lenhoso?

\section{MATERAL E MÉTODOS}

\section{Caracterização física e localização da área de estudo}

O estudo foi realizado em uma área de caatinga (com fisionomia arbórea à arbustivaarbórea) localizada no Parque Estadual da Mata da Pimenteira (Decreto $n^{\circ} 37.823$, de 20/01/2012), município de Serra Talhada, Pernambuco, entre novembro de 2009 e novembro de 2010. A área apresenta altitudes variando de 500 a $700 \mathrm{~m}$ (FERRAZ et al., 1998). Os solos predominantes daquela região são luvissolos (fase pedregosa) e neossolos litólicos eutróficos (JACOMINE et al., 1973). A precipitação média anual é de aproximadamente $650 \mathrm{~mm}$, com ampla variação ao longo do tempo, e temperatura média de $26^{\circ} \mathrm{C}$, com pequenas variações ao longo do tempo (MELO, 1988). A precipitação total observada no período de realização do estudo foi de $734 \mathrm{~mm}$ (dado obtido a partir do site do Instituto Nacional de Pesquisas Espaciais (http://www6.cptec.inpe.br/proclima/))

\section{Coleta e análise dos dados de vegetação}

Para o levantamento quantitativo das plantas lenhosas foi utilizado o método de amostragem de Parcelas Permanentes de Monitoramento (PPM) com dimensões de $20 \times 50 \mathrm{~m}$ subdivididas em 10 parcelas de $10 \times 10 \mathrm{~m}$. A amostragem foi dividida em dois ambientes: no ambiente I foram alocadas quatro PPM distanciadas cerca de cinco metros da margem do curso d'água (sendo a parte dos 20 $\mathrm{m}$ posicionada paralelamente ao curso d'água), quando a fisionomia do componente lenhoso é mais densa e no ambiente II, distante cerca de $100 \mathrm{~m}$ do curso d'água, quando a fisionomia é mais aberta, foram distribuídas as outras quatro PPM. Em cada parcela, todos os indivíduos lenhosos (arbustivoarbóreos) vivos com diâmetro do caule ao nível do solo $(\mathrm{DNS}) \geq$ a $3 \mathrm{~cm}$ e altura total $\geq 1 \mathrm{~m}$ foram 
marcados com uma plaqueta de alumínio numerada e foram anotados os dados de altura total e DNS. Em um dos vértices de cada parcela foi plotada uma subparcela de $2 \times 2 \mathrm{~m}$ para medir a altura e diâmetro dos indivíduos das espécies amostradas no componente lenhoso (arbustivo-arbóreo) com DNS entre 0,5 e $2,9 \mathrm{~cm}$ (regeneração).

Foram realizadas viagens periódicas à área de estudo para coleta de material reprodutivo das espécies amostradas nas parcelas. $\mathrm{O}$ material coletado foi herborizado, seguindo as técnicas usuais de preparação, secagem e montagem das exsicatas e posteriormente identificado por meio de literatura especializada, comparação com as exsicatas depositadas nos Herbário Sérgio Tavares (UFRPE) e Dárdano de Andrade Lima (IPA), além de envio para especialistas de outras instituições brasileiras.

Para análise dos dados do levantamento foram calculados os descritores fitossociológicos gerais da comunidade tanto do componente lenhoso como para a regeneração (densidade, valores de diâmetros e alturas médios e máximos, e, área basal total e por hectare, dos diferentes ambientes) e avaliada a distribuição de indivíduos em histogramas com intervalo fixo de $3 \mathrm{~cm}$ para diâmetro e $1 \mathrm{~m}$ para altura, para o componente lenhoso, considerando a amostra como um todo. Para distribuição dos indivíduos regenerantes considerou-se intervalos de $0,4 \mathrm{~cm}$ e $0,9 \mathrm{~cm}$ para diâmetros e alturas, respectivamente. Os dados de densidade, índices de diversidade de Shannon (H', em nats./ind.) e equabilidade de Pielou (J') foram calculados com o uso do programa Mata Nativa 2.0. Os índices de diversidade de Shannon entre os ambientes foram comparados pelo teste de $\mathrm{t}$ de Hutcheson (ZAR, 1999). A similaridade florística (matriz de presença-ausência) entre os ambientes foi analisada pelo índice de Sørensen. Foi calculado ainda, o número de indivíduos perfilhados para os dois ambientes e estratos.

Após verificação da normalidade pelo teste Kolmogorov-Smirnov, os valores de densidade, área basal, diâmetro médio e máximo, altura média e máxima por parcela foram comparados entre os ambientes. Nos dados com distribuição normal, foi empregada a análise de variância (ANOVA) de um critério, sendo as diferenças consideradas conclusivas pelo teste $\mathrm{F}$ a $5 \%$ de probabilidade (ZAR, 1999). Dados sem distribuição normal foram analisados pelo teste não paramétrico de Mann-Whitney. Para verificar se houve diferença na distribuição dos indivíduos nas classes diamétricas, entre os ambientes, utilizou-se o teste de Kolmogorov-Smirnov (2xn), para duas amostras independentes, em nível de $5 \%$. Todas as análises foram realizadas com o auxílio do programa Bioestat 2.0. A suficiência amostral foi avaliada por meio do procedimento de Regressão Linear com Resposta em Platô (REGRELRP), pelo Sistema para Análises Estatísticas (SAEG) versão 5.0.

\section{Levantamento e análise das variáveis ambientais}

Para caracterização físico-química do solo foram coletadas por meio de tradagem cinco amostras de cada parcela de $20 \times 50 \mathrm{~m}$ a uma profundidade de $0-20 \mathrm{~cm}$. As cinco amostras de solo foram homogeneizadas e foi retirada uma única amostra composta de $0,5 \mathrm{~kg}$ de solo. As amostras foram acondicionadas em sacos plásticos de $1000 \mathrm{~mL}$ que foram devidamente identificados e levados para o Laboratório de Química do Solo da Universidade Federal Rural de Pernambuco, onde se efetuaram as análises físico-químicas das mesmas, seguindo o protocolo da EMBRAPA (1997). As variáveis de solo obtidas foram: $\mathrm{pH}, \mathrm{P}, \mathrm{K}+, \mathrm{Ca}++$, $\mathrm{Mg}++, \mathrm{Al}+++$, porcentagem de areia, silte, argila e matéria orgânica.

Foram avaliadas ainda rochosidade e pedregosidade de cada parcela, utilizando-se como referência o documento $n^{\circ} 11$ da EMBRAPA (1988). Com o auxílio de um sistema de posicionamento global (GPS) foram obtidas as coordenadas de cada vértice das parcelas de $10 \times 10 \mathrm{~m}$ e a partir dessas coordenadas foram extraídas as seguintes variáveis topográficas: distância até a margem do curso d'água, obtida da diferença entre a altitude média dos quatro vértices da parcela e a altitude da margem do curso d'água; e declividade média, obtida da média da declividade dos quatro lados das parcelas. As médias das variáveis pedológicas e topográficas por parcela foram comparadas entre os ambientes usando os mesmos testes utilizados para comparação dos parâmetros fisionômicos e estruturais citados anteriormente.

Para analisar as correlações entre as variações na abundância de espécies (componente lenhoso e regenerante) por parcela com variáveis ambientais foi utilizada uma análise de correspondência canônica (CCA) utilizando o programa PC-ORD for Windows versão 4.14 (MCCUNE; MEFFORD, 1999). A matriz de abundância das espécies foi constituída do número de indivíduos por parcela das 
espécies que apresentaram mais de cinco indivíduos na amostra total. De acordo com as recomendações de Ter Braak (1995), os valores de abundância foram transformados $(\log 10(\mathrm{a}+1))$ para compensar os desvios causados por alguns poucos valores muito elevados.

A matriz de variáveis ambientais incluiu, a princípio, por parcela, pedregosidade, rochosidade, declividade, distância vertical e as variáveis químicas e texturais dos solos. Após a realização de uma CCA preliminar, foram retiradas da análise as variáveis ambientais redundantes ou que apresentaram uma baixa correlação $(<0,4)$ com os eixos 1 e 2. A CCA final foi processada com as variáveis fortemente correlacionadas com os eixos de ordenação. Para o componente lenhoso, as variáveis que permaneceram na análise foram $\mathrm{K}, \mathrm{Ca}++$, argila, pedregosidade, declividade e distância vertical, enquanto para regeneração foram $\mathrm{K}, \mathrm{Ca}++$, argila, declividade e distância vertical. Em seguida, foi realizado um teste de Análise de Espécies Indicadoras para verificar a significância das espécies como indicadoras dos ambientes para ambos os estratos (DUFRÊNE; LEGENDRE, 1997), agregada ao teste de Monte Carlo.

\section{RESULTADOS E DISCUSSÃO}

\section{Suficiência Amostral}

A curva cumulativa de espécies para ambos os estratos e ambientes em relação à área amostral levantada, indicou tendência à estabilização à medida que se aumentou o número de parcelas. A maioria das espécies no componente lenhoso (95\% versus $77 \%$ ) foi encontrada em metade das parcelas com valores de $\mathrm{R}^{2} \quad\left(\mathrm{R}^{2}=88,28\right.$ versus $\left.\mathrm{R}^{2}=86,12\right)$ nos ambientes I e II, respectivamente, enquanto para regeneração (62,5\% versus $65,22 \%$ das espécies) com $\left(\mathrm{R}^{2}=96,58\right.$ versus 97,62) (Figura 1). De acordo com Muller-Dumbois e Ellenberg (1974), este comportamento da curva sugere que o estudo teve uma ampla representatividade florística.
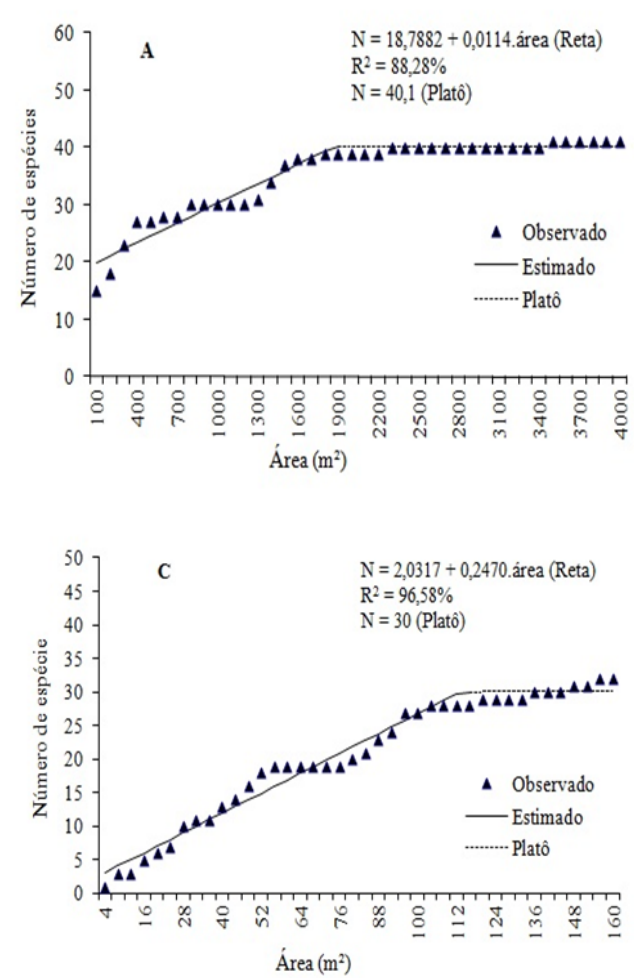
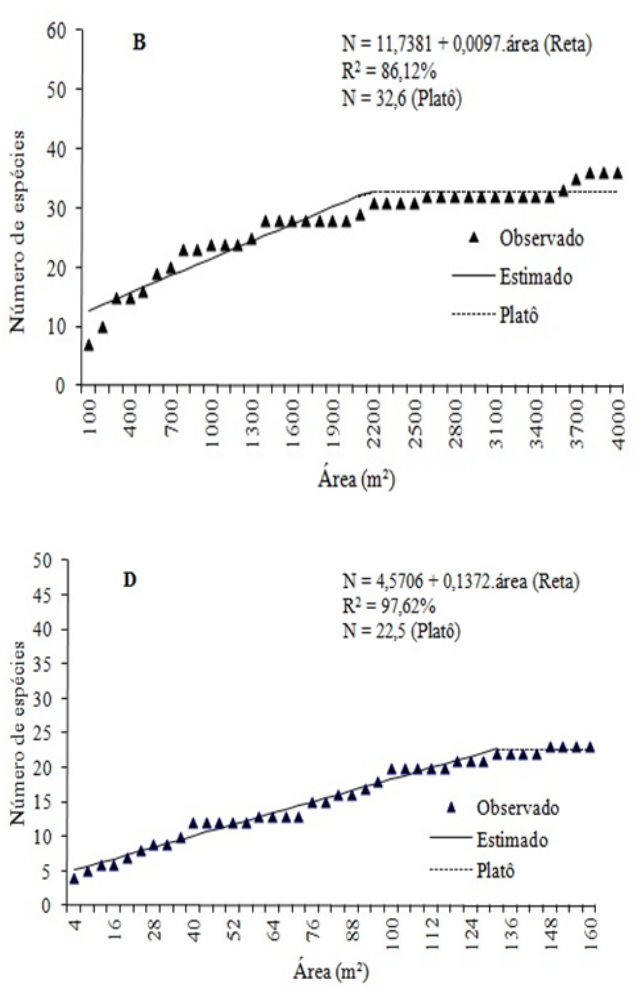

FIGURA 1: Suficiência amostral da área de estudo para o componente lenhoso dos ambientes I (A) e II (B) e para regeneração dos ambientes I (C) e II (D). Os valores de N (Platô) correspondem ao número de espécies estimado no qual inicia o platô de estabilização das curvas.

FIGURE 1: Sample sufficiency of the study area from woody component of the environments I (A) and II (B) and regeneration of environments I (C) and II (D). The N values (Plateau) correspond to the estimated number of species in which the plateau of the curves' stabilization starts. 
Fisionomia do componente lenhoso e sua
regeneração

Os ambientes I e II apresentaram diferença significativa quanto ao número de indivíduos $(4047,5$ versus 3332,5 ind.ha $\left.^{-1}\right)$ e área basal $(23,6$ versus 17,6 $\mathrm{m}^{2}$. ha $\left.{ }^{-1}\right)$, com valores significativamente superiores no ambiente I. Padrão oposto ao da regeneração em que o número de indivíduos foi maior no ambiente II 9187,5 versus 10937,5 ind.ha $^{-1}$, respectivamente (Tabela 1).

Sabe-se que a densidade e a área basal das florestas tropicais apresentam variações de acordo com as condições de solo, disponibilidade hídrica, luminosidade, temperatura, interações biológicas, condições de micro-habitat, estádios de regeneração, entre outras (GOULD; GONZÀLEZ; CARRERO, 2006; POWERS et al., 2009). A disponibilidade hídrica tem sido apontada como um dos principais fatores, que afetam as comunidades vegetais em áreas submetidas à forte sazonalidade climática (TREJO; DIRZO, 2002; SEGURA et al., 2003), como ocorre em ambientes de vegetação de caatinga (ALCOFORADO-FILHO; SAMPAIO; RODAL, 2003; ANDRADE et al., 2009).

Os valores de densidade e área basal sugerem que ambientes próximos a cursos d'água têm maior biomassa, semelhante ao relatado em outros estudos em áreas de caatinga que analisaram a mesma heterogeneidade de habitat avaliada neste estudo (RODAL; COSTA; LINS-E-SILVA, 2008a), ou ainda, heterogeneidade de habitat relacionada a diferentes gradientes de umidade influenciados pela altitude (FERRAZ; RODAL; SAMPAIO, 2003), os quais também demonstraram forte influência nos descritores estruturais de densidade (3050 a 6535 ind.ha' $\left.{ }^{-1}\right)$ e área basal $\left(30,6\right.$ a $56,7 \mathrm{~m}^{2}$. ha' $\mathrm{h}^{-1}$.

Valores de densidade e área basal superiores e inferiores aos encontrados neste estudo foram registrados em outras áreas de caatinga (FERRAZ; RODAL; SAMPAIO, 2003; PEREIRA et al., 2001; ALCOFORADO-FILHO; SAMPAIO; RODAL, 2003; RODAL; COSTA; LINS-eSILVA, 2008a; RODAL; MARTINS; SAMPAIO, 2008b). Alcoforado-Filho, Sampaio e Rodal (2003) ressaltaram ainda, que parte da amplitude encontrada entre os diversos estudos em áreas de caatinga pode relacionar-se à disponibilidade de água nos diferentes ambientes, ou ainda ao regime de manejo dessas áreas.

Em termos de diâmetros não houve diferenças nos ambientes, tanto no componente lenhoso como para regeneração (Tabela 1). $\mathrm{O}$ ambiente I diferiu significativamente pelas suas maiores alturas média $(4,7$ versus $4,2 \mathrm{~m})$ e máxima $(9,3$ versus $7,0 \mathrm{~m})$. Padrão oposto foi verificado para regeneração, na qual a altura média foi significativamente superior no ambiente II (Tabela 1).

No componente lenhoso, a maioria dos indivíduos ocorreu na primeira classe de diâmetro $(3,0-6,0 \mathrm{~cm})$, com $62,9 \%$ das plantas do ambiente

TABELA 1: Descritores quantitativos do componente lenhoso e da regeneração nos ambientes I e II da Mata da Pimenteira, em Serra Talhada, Pernambuco, Brasil.

TABLE 1: Quantitative descriptors of the woody component and regeneration in the environments I and II of the Mata of Pimenteira, in "Serra Talhada", Pernambuco State, Brazil.

\begin{tabular}{lcccc}
\hline \multicolumn{1}{c}{ Parâmetros } & \multicolumn{2}{c}{ Componente Lenhoso } & \multicolumn{2}{c}{ Regeneração } \\
\hline Densidade (ind./ha) & $\mathrm{I}$ & $\mathrm{II}$ & $\mathrm{I}$ & $\mathrm{II}$ \\
Área Basal Total & $4047,5 \mathrm{a}$ & $3332,5 \mathrm{~b}$ & $9187,5 \mathrm{~b}$ & $10937,5 \mathrm{a}$ \\
Área Basal (m²/ha) & $9,4 \mathrm{a}$ & $7,0 \mathrm{~b}$ & $0,03 \mathrm{a}$ & $0,04 \mathrm{a}$ \\
Diâmetro Médio (cm) & $23,6 \mathrm{a}$ & $17,6 \mathrm{~b}$ & $1,9 \mathrm{a}$ & $2,5 \mathrm{a}$ \\
Diâmetro Máximo (cm) & $6,7 \mathrm{a}$ & $6,6 \mathrm{a}$ & $1,4 \mathrm{a}$ & $1,5 \mathrm{a}$ \\
Altura Média (m) & $26,8 \mathrm{a}$ & $23,8 \mathrm{a}$ & $1,9 \mathrm{a}$ & $2,4 \mathrm{a}$ \\
Altura Máxima (m) & $4,7 \mathrm{a}$ & $4,2 \mathrm{~b}$ & $1,6 \mathrm{~b}$ & $1,9 \mathrm{a}$ \\
Índice de Shannon-Wiener (H') & $9,3 \mathrm{a}$ & $7,0 \mathrm{~b}$ & $2,4 \mathrm{a}$ & $2,8 \mathrm{a}$ \\
Equabilidade ( $\left.\mathrm{J}^{\prime}\right)$ & $2,80 \mathrm{a}$ & $2,53 \mathrm{~b}$ & $2,96 \mathrm{a}$ & $2,46 \mathrm{~b}$ \\
Perfilhamento (n ${ }^{\circ}$ de indivíduos) & $0,75 \mathrm{a}$ & $0,71 \mathrm{~b}$ & $0,85 \mathrm{a}$ & $0,78 \mathrm{~b}$ \\
\hline
\end{tabular}

Em que: Médias seguidas de letras diferentes representam diferença estatística significativa entre os ambientes $(p \leq 0,05)$. 
I, o qual diferiu significativamente do ambiente II, que esteve representado por $50,7 \%$ dos indivíduos $(\mathrm{p} \leq 0,05)$. Por outro lado, o ambiente I apresentou uma maior concentração de indivíduos nas classes de maior diâmetro. O número de indivíduos decresceu com o aumento das classes diamétricas, gerando um gráfico que se assemelha com a forma de "J invertido", ou seja, elevada concentração de indivíduos nas primeiras classes e redução acentuada no sentido das demais classes (Figura 2A). A maior concentração de indivíduos no componente lenhoso ocorreu no intervalo de classe de altura de (4,1-5 m) nos dois ambientes, $27,1 \%$ (I) e 26,2\% (II) (Figura 2B). No entanto, o ambiente I diferiu do ambiente II com maior número de indivíduos no intervalo de classe de altura de (1,0-2 m) e em alturas superiores a $5 \mathrm{~m}$, sendo esta diferença observada até $8 \mathrm{~m}$ de altura.

Esse padrão parece confirmar o relatado em florestas tropicais, no qual independentemente do estágio de desenvolvimento dessas florestas, há uma tendência a maior concentração de indivíduos nas classes de menores diâmetros e alturas (CONDIT et al., 1998; DEWALT; SCHNITZER; DENSLOW, 2003). O fato do ambiente I diferir significativamente em função do maior número de indivíduos nas classes de maior diâmetro e altura e valores de altura média e máxima superiores aos do ambiente II, pode relacionar-se à baixa disponibilidade de água no ambiente II, resultando em baixa produtividade líquida. Consequentemente, promovendo uma maior restrição no desenvolvimento das árvores, uma vez que estas respondem às variações nas propriedades do solo e seus efeitos na maior ou menor disponibilidade de nutrientes e água com alterações na estrutura e composição da vegetação (POWERS et al., 2009).

Além dessa questão, pode-se ainda pensar que o efeito da elevada densidade do componente lenhoso, verificada no ambiente I, poderia ser responsável pelo maior crescimento em altura das árvores, possivelmente pela maior competição por luz decorrente do maior fechamento das copas neste ambiente. De acordo com King (1990), indivíduos estabelecidos em ambientes mais úmidos tendem a alcançar valores de altura superior aos de ambientes secos, provavelmente devido à grande cobertura das copas das árvores e ausência de deciduidade nas florestas úmidas, as quais reduzem a intensidade de luz e, o que obriga os indivíduos a crescerem em altura para alcançar o estrato superior da floresta. Todavia, este sombreamento não ocorre em áreas mais secas, portanto, não justifica o maior crescimento em altura observado no ambiente I.

Mais da metade dos indivíduos da regeneração nos ambientes I e II estiveram presentes nas duas primeiras classes de diâmetro $(0,5-0,9$ $\mathrm{cm} ; 0,91-1,31 \mathrm{~cm})$, representando cerca de $60 \%$ e $70 \%$ do total dos indivíduos, respectivamente (Figura 3A). A comparação dos dois ambientes em termos das classes de diâmetro e altura revelou que o ambiente II foi significativamente superior, em alguns intervalos de classes, em ambos os aspectos (Figuras 3A e 3B). Tanto para o componente
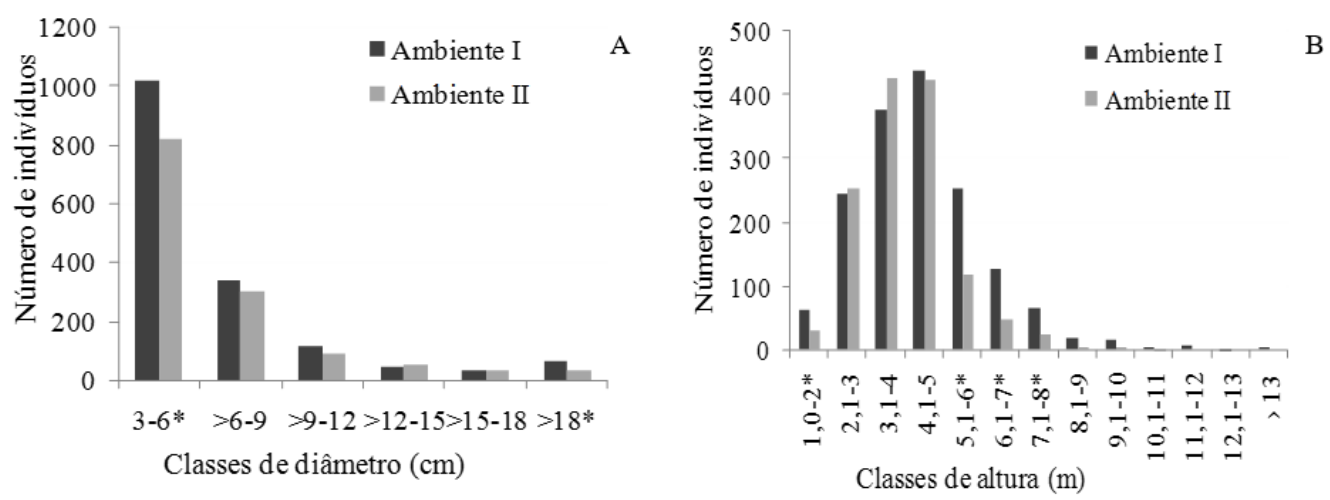

FIGURA 2: Distribuição em classes de diâmetro e altura dos indivíduos do componente lenhoso (A e B) nos ambientes (I e II) da Mata da Pimenteira, em Serra Talhada, Pernambuco, Brasil. Colunas com * na mesma classe diferem entre si $(p \leq 0,05)$.

FIGURE 2: Distribution of diameter and height classes of individuals of the woody component (A and B) in the environment (I and II) of the Mata of Pimenteira, in "Serra Talhada", Pernambuco State, Brazil. Columns with $*$ in the same class differ from each other $(\mathrm{p} \leq 0,05)$. 
lenhoso como para regeneração, o ambiente II foi significativamente superior em número de indivíduos perfilhados (300 versus 452,5 ind.ha ${ }^{-1}$ ) e (312,5 versus 2125 ind ha $\left.^{-1}\right)$, de acordo com o Teste $\mathrm{t}, \mathrm{p}=0,006$ e $\mathrm{p}=0,002$, respectivamente (Tabela 1 ).

Contrariamente ao esperado, a diferença entre os ambientes, observada na regeneração, em termos de maior densidade e crescimento em altura, poderia ser explicada pela influência da maior disponibilidade de luminosidade no ambiente II, criando micro-habitat favorável para desencadear a germinação das sementes e desenvolvimento das plântulas. Nesse sentido, Wirth, Weber e Ryel (2001) afirmaram que a abertura do dossel em florestas tropicais promove o crescimento e germinação das sementes de plantas lenhosas na regeneração. Bianchini, Pimenta e Santos (2009) relataram ainda, que as variações da luminosidade podem influenciar indiretamente os parâmetros estruturais e fisionômicos da regeneração, causando alterações dentre outros fatores, na temperatura e disponibilidade de umidade.

Outro fato, que possivelmente está associado à maior entrada de luz para regeneração do ambiente II, foi a grande quantidade de indivíduos perfilhados (aproximadamente sete vezes maior) amostrados, comparando-se à regeneração do ambiente I.

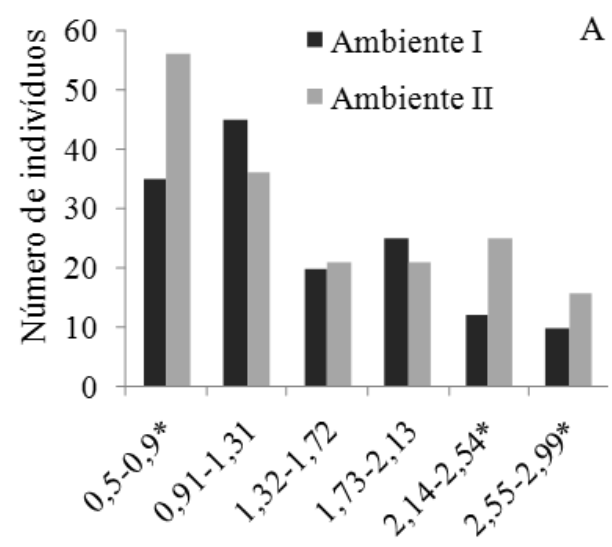

Classes de diâmetro $(\mathrm{cm})$

\section{Estrutura do componente lenhoso e sua regeneração}

As 50 espécies identificadas no conjunto das áreas estudadas ficaram distribuídas em 24 famílias e 41 gêneros, com destaque para Fabaceae e Euphorbiaceae nos diferentes estratos e ambientes estudados (Tabela 2). Fabaceae esteve representada por quinze espécies e Euphorbiaceae por sete, perfazendo $44 \%$ do total de espécies amostradas. Estas famílias estiveram entre as de maior riqueza em vários levantamentos realizados em áreas de caatinga, conforme atestam os trabalhos de Pereira et al. (2001), Gomes, Rodal e Melo (2006) e Andrade et al., (2009). Os índices de diversidade de Shannon-Wiener (H') e de equabilidade (J) diferiram entre os ambientes com valores de 2,80 nats.ind ${ }^{-1} \mathrm{e}$ 0,75 e 2,53 nats.ind ${ }^{-1}$ e 0,71 nos ambientes I e II, respectivamente (Tabela 1 ).

Conforme a Tabela 2, do total de 45 espécies amostradas no componente lenhoso nos ambientes I e II, 41 foram registradas no ambiente I e 36 no ambiente II, com 32 compartilhadas o que corresponde a $71 \%$ do total. Mais de $50 \%$ das espécies amostradas no componente lenhoso dos dois ambientes estiveram presentes na regeneração, sendo nove exclusivas do ambiente I e três do II. A análise de similaridade florística mostrou que os ambientes são muito semelhantes com índice de similaridade de Sørensen $(0,83)$. Do total de espécies registradas nesse componente, cinco foram

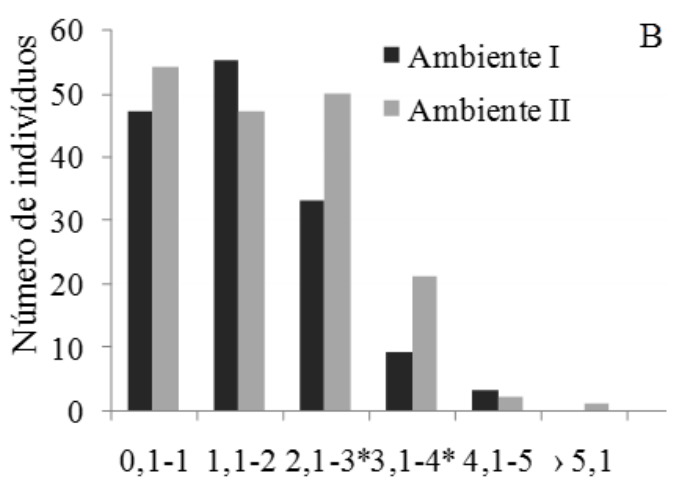

Classes de altura (m)

FIGURA 3: Distribuição em classes de diâmetro e altura dos indivíduos da regeneração (A e B) nos ambientes (I e II) da Mata da Pimenteira, em Serra Talhada, Pernambuco, Brasil. Colunas com * na mesma classe diferem entre si $(\mathrm{p} \leq 0,05)$.

FIGURE 3: Distribution of diameter and height classes of individuals of regeneration (A and B) in the environment (I and II) of the Mata of Pimenteira, in "Serra Talhada", Pernambuco State, Brazil. Columns with * in the same class differ from each other $(\mathrm{p} \leq 0,05)$. 
consideradas indicadoras do ambiente I e quatro do ambiente II (Tabela 2).
Independentemente do ambiente, as populações mais abundantes foram Croton

TABELA 2: Número de indivíduos das famílias e das espécies do componente lenhoso e da regeneração na Mata da Pimenteira, em Serra Talhada, Pernambuco, Brasil, em ordem decrescente do número total de indivíduos por família. *Ambiente e componente em que a espécie foi indicadora.

TABLE 2: Number of individuals of families and species of the woody component and regeneration in the Mata of Pimenteira, in "Serra Talhada", Pernambuco State, Brazil, in descending order of total number of individuals per family. *Environment and component where the species was indicator.

\begin{tabular}{|c|c|c|c|c|}
\hline \multirow{2}{*}{ Espécies/Famílias } & \multicolumn{2}{|c|}{ Componente Lenhoso } & \multicolumn{2}{|c|}{ Regeneração } \\
\hline & Ambiente I & Ambiente II & Ambiente I & Ambiente II \\
\hline EUPHORBIACEAE & 568 & 580 & 32 & 55 \\
\hline Croton blanchetianus Baill. & 145 & $370^{*}$ & 13 & 25 \\
\hline Croton rhamnifolioides Pax. \& K. Hoffm. & $254 *$ & 77 & 7 & 6 \\
\hline Ditaxis desertorum Pax \& K. Hoffm. & 1 & - & 1 & $12 *$ \\
\hline Jatropha mollissima (Pohl.) Baill. & 53 & 64 & 2 & 2 \\
\hline Manihot epruinosa Pax \& K. Hoffm. & 85 & 66 & 4 & 10 \\
\hline Sapium glandulosum (L.) Morong & 7 & 3 & - & - \\
\hline Sebastiana macrocarpa Mull. Arg. & 23 & - & 5 & - \\
\hline FABACEAE & 419 & 447 & 36 & 61 \\
\hline Amburana cearensis (Allemão) A. C. Sm. & 6 & 6 & 2 & 1 \\
\hline Anadenanthera colubrina (Vell.) Brenan & 37 & 46 & 3 & 7 \\
\hline Bauhinia cheilantha (Bong) D. Dietr. & 214 & 210 & 15 & $41 *$ \\
\hline Chloroleucon mangense Britton \& Rose & 4 & 1 & - & 1 \\
\hline Lutzelburgia auriculata (Allemão) Ducke & 1 & - & - & - \\
\hline Libidibia ferrea (Mart. ex. Tul.) L. P. Queiroz & 11 & 2 & 1 & - \\
\hline Mimosa ophtalmocentra Mart. ex. Benth & 3 & - & 1 & - \\
\hline Mimosa tenuiflora (Willd.) Poir. & 12 & $56^{*}$ & 5 & 1 \\
\hline $\begin{array}{l}\text { Paraptadenia zehntneri (Harms) M. P. Lima \& H. C. } \\
\text { Lima }\end{array}$ & $26^{*}$ & 5 & 4 & 1 \\
\hline Piptadenia stipulacea (Benth.) Ducke & 4 & $23 *$ & - & - \\
\hline Poincianella pyramidalis (Tul.) L. P. Queiroz & 99 & 96 & 3 & 8 \\
\hline Senna macranthera (Collad.) H. S. Irwin \& Barneby & 2 & 1 & - & 1 \\
\hline Senna spectabilis (DC.) H. S. Irwin \& Barneby & - & 1 & - & - \\
\hline Fabaceae 1 & - & - & 1 & - \\
\hline Fabaceae 2 & - & - & 1 & - \\
\hline APOCYNACEAE & 323 & 88 & 7 & 2 \\
\hline Aspidosperma pyrifolium Mart. & 60 & 63 & 4 & 2 \\
\hline Aspidosperma cuspa S. F. Blake ex Pittier & $263 *$ & 25 & 3 & - \\
\hline ANNONACEAE & 69 & 89 & 18 & 5 \\
\hline Rollinia leptopetala R. E. Fr. & 69 & 89 & 18 & 5 \\
\hline SAPINDACEAE & 45 & 7 & 2 & - \\
\hline Allophylus quercifolius Radlk. & $45 *$ & 7 & 2 & - \\
\hline RUBIACEAE & 37 & 1 & 6 & 1 \\
\hline Guettarda angelica Mart. ex Müll. Arg. & $37 *$ & 1 & 6 & 1 \\
\hline COMBRETACEAE & 35 & 6 & 10 & 2 \\
\hline Combretum pisonioides Taub. & 35 & 6 & 10 & 2 \\
\hline MALVACEAE & 27 & 21 & - & - \\
\hline Ceiba glaziovii (Kuntze) K. Schum. & - & 1 & - & - \\
\hline $\begin{array}{l}\text { Pseudobombax marginatum (A. St.-Hil., A. Juss. \& } \\
\text { Cambess.) A. Robyns }\end{array}$ & 25 & 20 & - & - \\
\hline Helicteres mollis K. Schum. & 2 & - & - & - \\
\hline
\end{tabular}


TABELA 2: Continuação...

TABLE 2: Continued...

\begin{tabular}{|c|c|c|c|c|}
\hline \multirow{2}{*}{ Espécies/Famílias } & \multicolumn{2}{|c|}{ Componente Lenhoso } & \multicolumn{2}{|c|}{ Regeneração } \\
\hline & Ambiente I & Ambiente II & Ambiente I & Ambiente II \\
\hline CAPPARACEAE & 20 & 11 & 3 & 5 \\
\hline Cynophalla flexuosa (L.) J. Presl & 18 & 11 & 3 & 5 \\
\hline Neocalyptrocalyx longifolium (Cornejo) Mart. \& Iltis & 2 & - & - & - \\
\hline SALICACEAE & 19 & 8 & - & 1 \\
\hline Prockia crusis L. & 19 & 8 & - & 1 \\
\hline ANACARDIACEAE & 17 & 11 & 1 & 1 \\
\hline Myracrodruon urundeuva Allemao & 12 & 8 & 1 & 1 \\
\hline Schinopsis brasiliensis Engl. & 5 & 2 & - & - \\
\hline Spondias tuberosa Arruda & - & 1 & - & - \\
\hline MALPHIGIACEAE & 11 & 2 & - & 2 \\
\hline Ptilochaeta bahiensis Turcz. & 11 & 2 & - & 2 \\
\hline BURSERACEAE & 7 & 48 & 1 & 10 \\
\hline Commiphora leptophloeos (Mart.) J. B. Gillett & 7 & $48^{*}$ & 1 & $10^{*}$ \\
\hline CARICACEAE & 6 & - & 1 & - \\
\hline Jacaratia corumbensis Kuntze & 6 & - & 1 & - \\
\hline RHAMNACEAE & 4 & 3 & - & - \\
\hline Ziziphus joazeiro Mart. & 4 & 3 & - & - \\
\hline BORAGINACEAE & 3 & - & 23 & 30 \\
\hline Varronia leucocephala (Moric.) J. S. Mill. & 3 & - & 23 & $30 *$ \\
\hline ERYTHROXYLACEAE & 3 & 3 & 3 & - \\
\hline Erythroxylum caatingae Plowman & 3 & 3 & 3 & - \\
\hline CACTACEAE & 2 & 2 & - & - \\
\hline Cereus jamacaru DC. & 2 & 2 & - & - \\
\hline NYCTAGINACEAE & 2 & 5 & 1 & - \\
\hline Guapira laxa (Netto) Furlan & 2 & 5 & 1 & - \\
\hline SOLANACEAE & 2 & - & - & - \\
\hline Solanum sp. & 2 & - & - & - \\
\hline BIGNONIACEAE & - & - & 1 & - \\
\hline Bignoniaceae 1 & - & - & 1 & - \\
\hline INDETERMINADA E & - & - & 1 & - \\
\hline Indeterminada $\mathrm{E}$ & - & - & 1 & - \\
\hline MYRTACEAE & - & - & 1 & - \\
\hline Eugenia punicifolia DC. & - & - & 1 & - \\
\hline VERBENACEAE & - & 1 & - & - \\
\hline Lantana camara L. & - & 1 & - & - \\
\hline TOTAL & 1619 & 1333 & 147 & 175 \\
\hline
\end{tabular}

blanchetianus, Croton rhamnifolioides, Jatropha mollissima, Manihot epruinosa, Anadenanthera colubrina, Bauhinia cheilantha, Poincianella pyramidalis, Aspidosperma pyrifolium e Rollinia leptopetala. Estas espécies representam cerca de $60 \%$ e $80 \%$ da densidade total dos ambientes I e II, respectivamente (Tabela 2). Dentre as espécies abundantes acima mencionadas apenas Croton blanchetianus e Croton rhamnifolioides diferiram em número de indivíduos entre os ambientes I e II, sendo que a primeira apresentou valor superior no ambiente II e Croton rhamnifolioides esteve presente com maior número de indivíduos no ambiente I.

Entre as espécies mais abundantes apenas
Croton rhamnifolioides, Jatropha mollissima, Manihot epruinosa e Anadenanthera colubrina diferiram entre os ambientes I e II em termos de diâmetros médios, enquanto Croton blanchetianus, Jatropha mollissima, Bauhinia cheilantha, Poincianella pyramidalis e Anadenanthera colubrina diferiram em altura média. Um fato que merece destaque foi a elevada densidade de Aspidosperma cuspa no componente lenhoso do ambiente I (Tabela 2), diferindo do ambiente II tanto em número de indivíduos como em altura média.

$\mathrm{Na}$ regeneração foram amostradas 36 espécies, sendo 32 no ambiente I e 23 no ambiente II (Tabela 2). Cerca de $84 \%$ das espécies da regeneração 
do ambiente I ocorreram no componente lenhoso do mesmo ambiente, contra $91 \%$ no ambiente II. Dentre as espécies registradas na regeneração dos dois ambientes, 13 foram exclusivas do ambiente I, enquanto apenas três (Senna macranthera, Prockia crusis e Ptilochaeta bahiensis) foram exclusivas do ambiente II, sendo 19 (54\%) comuns aos dois ambientes.

Os valores dos índices de diversidade de Shannon-Wiener (H') e de equabilidade (J) por parcela seguiram o mesmo padrão observado para o componente lenhoso, com valores significativamente maiores no ambiente I (Tabela1). Foi evidenciada uma alta similaridade florística entre os ambientes com índice de Sørensen $(0,69)$. Das 36 espécies registradas na regeneração, apenas quatro foram consideradas indicadoras do ambiente II, enquanto para o ambiente I não foi verificada distribuição significativamente diferenciada (Tabela 2).

Uma das possíveis causas relatadas na literatura, para explicar a maior ou menor diversidade encontrada nos diversos estudos quantitativos em áreas de caatinga, é a grande variação na disponibilidade hídrica observada nas diferentes áreas desse domínio (ANDRADE-LIMA, 1981; ALCOFORADO-FILHO; SAMPAIO; RODAL, 2003; AMORIM; SAMPAIO; ARAUJO, 2005), assim, pode-se supor que os maiores valores de diversidade observados no ambiente I, para os diferentes estratos, tenham sido influenciados pela proximidade do curso d'água, o que tornaria este ambiente mais favorável em termos de disponibilidade hídrica. Os valores de índice de diversidade de Shannon encontrados nos diferentes ambientes e estratos estiveram entre os maiores valores observados em áreas de caatinga (para revisão ver SAMPAIO, 1996).

Segura et al. (2003) observaram ao longo de um gradiente de umidade em florestas secas no México, um declínio na diversidade de espécies com a redução da disponibilidade hídrica. Padrão semelhante foi encontrado por Ferraz, Rodal e Sampaio (2003) ao longo de um gradiente de umidade em área no semiárido pernambucano. Ainda a esse respeito, Balvanera e Aguirre (2006) relataram que diferentes espécies ocupam diferentes partes da heterogeneidade espacial observada ao longo de gradientes de disponibilidade hídrica, e ainda, que muitas espécies podem ser excluídas de locais excessivamente secos nos quais a produtividade é muito baixa. $\mathrm{O}$ maior número de espécies exclusivas pode estar relacionado à maior heterogeneidade de habitat, o que foi verificado em ambos os estratos no ambiente I.

Dentre as espécies presentes na regeneração dos diferentes ambientes, Croton blanchetianus, Croton rhamnifolioides, Manihot epruinosa, Bauhinia cheilantha, Poincianella pyramidalis, Rollinia leptopetala, Ditaxis desertorum, Combretum pisonioides, Commiphora leptophloeos e Varronia leucocephala foram as mais abundantes, as quais juntas representaram $64 \%$ da densidade total do ambiente I e $85 \%$ do ambiente II (Tabela 2). Com exceção de Ditaxis desertorum e Varronia leucocephala, as demais espécies também foram as mais representativas em abundância no componente lenhoso dos diferentes ambientes. Este fato pode ser um indicativo de ausência de fatores que dificultem a renovação dessas populações por meios naturais.

Entre as populações mais abundantes acima mencionadas não foi evidenciada diferenças em número de indivíduos entre os ambientes. Em termos de valores médios de diâmetro e altura, Ditaxis desertorum e Varronia leucocephala apresentaram valores médios de diâmetro e altura superiores no ambiente II, enquanto Commiphora leptophloeos e Combretum pisonioides diferiram apenas em altura média, com valores superiores no ambiente I.

O padrão de distribuição do número de indivíduos por espécie nos ambientes estudados corrobora o encontrado para os diferentes tipos de caatinga, com elevada concentração da densidade em poucas espécies. Entretanto, o número de espécies do componente lenhoso nos dois ambientes pode ser considerado um dos mais elevados, quando comparados a outros levantamentos realizados em áreas de caatinga no Estado do Pernambuco (PEREIRA et al., 2001; FERRAZ; RODAL; SAMPAIO, 2003; ANDRADE et al., 2009; CAVALCANTI et al., 2009).

\section{Variáveis ambientais e distribuição das espécies}

A maioria das variáveis químicas e texturais dos solos não diferiram estatisticamente entre as parcelas correspondentes aos diferentes ambientes, com exceção dos teores de $\mathrm{Al}^{3+}$ que apresentou teores significativamente superiores no ambiente próximo ao curso d'água (Ambiente I). Com relação às demais variáveis edáficas analisadas, apenas a pedregosidade diferiu entre os ambientes I e II $(3,87$ versus $1,47 \%),(p \leq 0,05)$ (Tabela 3$)$. A alta similaridade florística na composição de espécies 
entre as comunidades reflete essa pouca diferença encontrada nas variáveis edáficas mensuradas entre os ambientes.

No componente lenhoso, os autovalores produzidos pela análise de correspondência canônica para os dois primeiros eixos de ordenação foram baixos 0,054 (eixo 1) e 0,025 (eixo 2) $(<0,5$ sensu TER BRAAK, 1995). A variância total explicada foi de apenas $49,2 \%$, indicando que as variáveis utilizadas contêm ainda muito 'ruído', ou seja, uma elevada proporção de variância não explicada, o que é muito comum em dados de vegetação (TER BRAAK, 1995). Apesar disso, a significância das relações espécie-ambiente não foi comprometida, já que a CCA produziu elevadas correlações espécieambiente, com autovalores nos dois primeiros eixos de 1,000 (eixo 1) e 1,000 (eixo 2). Além disso, o teste de permutação de Monte Carlo indicou que as correlações da abundância das espécies e variáveis ambientais foram altamente significativas $(\mathrm{p}<0,01)$ para os dois primeiros eixos.

As variáveis ambientais mais fortemente correlacionadas com o primeiro eixo foram, em ordem decrescente, pedregosidade, declividade e argila e, com o segundo eixo, $\mathrm{Ca}^{2+}$ e distância até o riacho (Tabela 3). Commiphora leptophloeos, Croton blanchetianus, Piptadenia stipulacea e Mimosa tenuiflora estiveram mais correlacionadas com as parcelas distante do curso d'água. Por outro lado, Croton rhamnifolioides, Sebastiana macrocarpa, Combretum pisonioides, Paraptadenia zehntneri e Guettarda angelica apresentaram maior correlação com as parcelas do ambiente próximo ao curso d'água.

$\mathrm{Na}$ análise de CCA da regeneração, os autovalores para os dois primeiros eixos foram baixos 0,294 (eixo 1) e 0,202 (eixo 2) e a variância total explicada foi de apenas 58,4\%. Essa baixa correlação (elevada variância remanescente) é esperadana análise de ordenação de dados ecológicos, devido à complexidade dos fatores envolvidos na determinação da composição florística e estrutural das formações vegetais (DALANESI; OLIVEIRAFILHO; FONTES, 2004). As correlações entre abundância das espécies e as variáveis ambientais foram altas 0,999 (eixo 1) e 0,977 (eixo 2) e significativamente correlacionadas para os dois primeiros eixos, pelo teste de permutação de

TABELA 3: Variáveis ambientais amostradas nos dois ambientes, com os valores das correlações com os dois primeiros eixos da análise de correspondência canônica (CCA), da Mata da Pimenteira, em Serra Talhada, Pernambuco, Brasil. Os valores correspondem às médias seguidas do desvio padrão.

TABLE 3: Environmental variables sampled in both environments, with the values of the correlations with the first two axes of canonical correspondence analysis (CCA), of the Mata of Pimenteira, in "Serra Talhada", Pernambuco State, Brazil. The values correspond to averages followed by standard deviation.

\begin{tabular}{|c|c|c|c|c|c|c|}
\hline \multirow{3}{*}{ Variáveis } & & & \multicolumn{4}{|c|}{ Correlações "inter-set" } \\
\hline & & & \multicolumn{2}{|c|}{ Componente lenhoso } & \multicolumn{2}{|c|}{ Regeneração } \\
\hline & Ambiente I & Ambiente II & Eixo 1 & Eixo 2 & Eixo 1 & Eixo 2 \\
\hline P Mehlich (mg.dm $\left.{ }^{-3}\right)$ & $6,59 \pm 2,41 a$ & $5,32 \pm 2,20 \mathrm{a}$ & - & - & - & - \\
\hline $\mathrm{K}^{+}\left(\mathrm{cmol}_{\mathrm{c}} \cdot \mathrm{dm}^{-3}\right)$ & $0,25 \pm 0,23 a$ & $0,33 \pm 0,13 \mathrm{a}$ & 0,294 & 0,434 & 0,457 & 0,377 \\
\hline $\mathrm{Ca}^{2+}\left(\mathrm{cmol}_{\mathrm{c}} \cdot \mathrm{dm}^{-3}\right)$ & $2,42 \pm 0,95 a$ & $1,88 \pm 0,97 \mathrm{a}$ & $-0,324$ & 0,666 & 0,253 & 0,677 \\
\hline $\mathrm{Mg}^{2+}\left(\mathrm{cmol}_{\mathrm{c}} \cdot \mathrm{dm}^{-3}\right)$ & $1,45 \pm 0,62 \mathrm{a}$ & $1,42 \pm 1,20 \mathrm{a}$ & - & - & - & - \\
\hline $\mathrm{Al}^{3+}\left(\mathrm{cmol}_{\mathrm{c}} \cdot \mathrm{dm}^{-3}\right)$ & $0,15 \pm 0,06 a$ & $0,05 \pm 0,04 b$ & - & - & - & - \\
\hline M.O (dag. $\left.\mathrm{kg}^{-1}\right)$ & $1,85 \pm 0,47 \mathrm{a}$ & $1,70 \pm 0,67 \mathrm{a}$ & - & - & - & - \\
\hline Areia $(\%)$ & $69,25 \pm 16,8 \mathrm{a}$ & $53,0 \pm 8,83 a$ & - & - & - & - \\
\hline Silte $(\%)$ & $13,0 \pm 12,88 \mathrm{a}$ & $31,75 \pm 16,1 \mathrm{a}$ & - & - & - & - \\
\hline Argila (\%) & $17,75 \pm 9,21 \mathrm{a}$ & $15,25 \pm 16,6 a$ & $-0,683$ & 0,373 & $-0,354$ & 0,670 \\
\hline Pedregosidade (\%) & $3,87 \pm 5,11 \mathrm{a}$ & $1,47 \pm 0,87 b$ & $-0,811$ & $-0,168$ & - & - \\
\hline Rochosidade (\%) & $1,22 \pm 5,29 a$ & $0,65 \pm 0,73 a$ & - & - & - & - \\
\hline Declividade (\%) & $10 \pm 9,60 \mathrm{a}$ & $8,25 \pm 7,70 \mathrm{a}$ & $-0,703$ & $-0,224$ & $-0,602$ & 0,396 \\
\hline Distância riacho (m) & $5 \pm 0,0 \mathrm{~b}$ & $100 \pm 0,0 \mathrm{a}$ & 0,482 & $-0,665$ & $-0,101$ & $-0,666$ \\
\hline
\end{tabular}

Em que: Médias seguidas de letras diferentes apresentam diferença estatística significativa $(p \leq 0,05)$ entre os ambientes. 
Monte Carlo $(\mathrm{p}<0,05)$. Sebastiana macrocarpa, Croton rhamnifolioides, Combretum pisonioides, Bauhinia cheilantha e Anadenanthera colubrina se encontraram em um grupo relacionado às parcelas do ambiente I, indicando alta correlação com as variáveis declividade e argila. Já Commiphora leptophloeos, Varronia leucocephala, Poincianella pyramidalis, Croton blanchetianus, Manihot epruinosa e Cynophalla flexuosa, tenderam a aumento de abundância com o aumento da distância do curso d'água.

A análise de correspondência canônica, apesar de ter mostrado uma clara separação entre as parcelas dos dois ambientes nos diferentes componentes, indicou a existência de um curto gradiente, ou seja, poucas substituições de espécies entre os dois extremos e predominância da variação nas abundâncias das espécies (TER BRAAK, 1995). Isso significa que as variáveis ambientais medidas explicaram, em parte, a variação da abundância das espécies.

\section{CONCLUSÕES}

Os resultados das comparações entre os parâmetros fisionômicos e estruturais corroboraram a hipótese de que a proximidade do curso d'água em áreas de caatinga influencia no comportamento da vegetação lenhosa e sua regeneração, em termos de densidade, crescimento em diâmetro e altura, riqueza e diversidade de suas populações. De modo geral, as maiores diferenças fisionômicas entre os ambientes parecem ser resultado principalmente da presença de populações com indivíduos de maiores alturas e diâmetros, sendo possível caracterizar a vegetação do ambiente I como uma vegetação de caatinga arbórea, enquanto o ambiente II como arbustivo-arbórea.

A heterogeneidade espacial dos fatores ambientais encontrada nessas áreas explicou parte das variações no comportamento das características florístico-estrutural da vegetação. As correlações significativas entre abundância de espécies e variáveis ambientais permitiram identificar tendências importantes e esse conjunto de tendências mostrou a importância das variações de fatores abióticos em pequena escala para se entender os padrões da vegetação ali existente. No entanto, a vegetação responde a diversos fatores bióticos e abióticos, os quais atuam em escalas de espaço e tempo diferentes, o que acaba dificultando a determinação de todos os processos que possam influenciar na organização das assembleias de plantas.

\section{REFERÊNCIAS BIBLIOGRÁFICAS}

ALCOFORADO-FILHO, F. G.; SAMPAIO, E. V. S. B.; RODAL, M. J. N. Florística e fitossociologia de um remanescente de vegetação caducifólia espinhosa arbórea em Caruaru, Pernambuco. Acta Botanica Brasilica, v. 17, p. 287-303, 2003.

AMORIM I. L.; SAMPAIO E. V. S. B.; ARAUJO E. L. Flora e estrutura da vegetação arbustivo-arbórea de uma área de caatinga do Seridó, RN, Brasil. Acta Botanica Brasilica, v. 19, p. 615-623, 2005.

ANDRADE, W. M. de. et al. Influência da precipitação na abundância de populações de plantas da caatinga. Revista de Geografia. RecifeUFPE, v. 26, p. 161-184, 2009.

ANDRADE-LIMA, D. The caatingas dominium. Revista Brasileira de Botânica, v. 4, p. 149-153, 1981.

BALVANERA, P.; AGUIRRE, E. Tree diversity, environmental heterogeneity, and productivity in a Mexican tropical dry forest. Biotropica, v. 38, p. 479-491, 2006.

BEGON, M.; TOWNSEND, C. R.; HARPER, J. L. Ecologia de indivíduos a ecossistemas. 4. ed. Porto Alegre: Artmed, 2007.

BIANCHINI, E.; PIMENTA, J. A.; SANTOS, F. A. M. Spatial and temporal variation in the canopy cover in a Tropical Semi-Deciduous Forest. Brazilian Archives of Biology and Technology, v. 44, p. 269-276, 2009.

CAVALCANTI, A. D. C. et al. Mudanças floríticas e estruturais, após cinco anos, em uma comunidade de caatinga no Estado de Pernambuco, Brasil. Acta Botanica Brasilica, v. 23, n. 3, p. 905-907, 2009. CONDIT, R. et al. Predicting population trends from size distributions: a direct test in a tropical tree community. The American Naturalist, v. 152, p. 495-509, 1998.

DALANESI, P. E.; OLIVEIRA-FILHO A. T.; FONTES, M. A. L. Flora e estrutura do componente arbóreo da floresta do Parque Ecológico Quedas do Rio Bonito, Lavras, MG e correlações entre a distribuição das espécies e variáveis ambientais. Acta Botanica Brasilica, v. 18, p. 737-757, 2004. DEWALT, S. J.; SCHNITZER, S. A.; DENSLOW, J. S. Density and diversity of lianas along a chronosequence in a central Panamanian lowland forest. Journal of Tropical Ecology, v. 16, p. 1-19, 2003. 
DUFRÊNE, M.; LEGENDRE, P. Species assemblages and indicator species: The need for a flexible asymmetrical approach. Ecological Monographs, v. 67, n. 3, p. 345-366, 1997.

EMBRAPA. Manual de métodos de análise de solos. Brasília: EMBRAPA, 1997. 212 p.

Serviço Nacional de Levantamento e Conservação de Solos. Critérios para distinção de classes de solos e de fases de unidades de mapeamento. Rio de Janeiro: EMBRAPA-SNLCS, 1988. $312 \mathrm{p}$.

FERRAZ, E. M. N.; RODAL, M. J. N.; SAMPAIO, E. V. S. B. Physiognomy and structure of vegetation along na altitudinal gradient in the semi-arid region of northeastern Brazil. Phytocoenologia, v. 33, p. 71-92, 2003.

et al. Composição florística em trechos de vegetação de caatinga e brejo de altitude na região do Vale do Pajeú, Pernambuco. Revista Brasileira de Botânica, v. 21, p. 7-15, 1998.

GOMES, A. P. S.; RODAL, M. J. N.; MELO, A. L. Florística e fitogeografia da vegetação arbustiva subcaducifólia da Chapada de São José, Buíque, PE, Brasil. Acta Botanica Brasilica, v. 20, p. 37-48, 2006.

GOULD, W. A.; GONZÀLEZ, G.; CARRERO, R. G. Structure and composition of vegetation along an elevational gradient in Puerto Rico. Journal of Vegetation Science, v. 17, p. 653-664, 2006.

JACOMINE, P. K. T. et al. Levantamento exploratório - reconhecimento de solos do Estado de Pernambuco. Recife: EMBRAPA - Divisão de Pesquisa Pedológica, 1973. 359 p.

KING, D. A. The adaptive significance of tree height. American Naturalist, v. 135, p. 809-828, 1990.

McCUNE, B.; MEFFORD, M. J. PC-ORD version 4.0., multivariate analysis of ecological data, Users guide. Glaneden Beach: MjM Software Design, 1999.

MELO, N. Áreas de exceção da Paraíba e dos Sertões de Pernambuco. Recife: SUDENE, 1988. $321 \mathrm{p}$.

MUELLER-DOMBOIS, D.; ELLENBERG, $\mathrm{H}$. Aims and methods of vegetation ecology. New York: John Willey \& Sons, 1974. 512 p.

MURPHY, P. G.; LUGO, A. E. Dry forests of Central America and Caribbean islands. In: BULLOCK, S. H; MOONEY, H. A.; MEDINA (Eds.). Seasonally dry tropical forests. Cambridge: Cambridge University Press, 1995. p. 9-34.
PEREIRA, I. M. et al. Regeneração Natural em um remanescente de caatinga sob diferentes níveis de perpetuação, no Agreste paraibano. Acta Botanica Brasilica, v. 15, p. 413-426, 2001.

POWERS, J. S. et al. Diversity and structure of regenerating tropical dry forests in Costa Rica: Geographic patterns and environmental drivers. Forest Ecology and Management, v. 258, p. 959-970, 2009.

RODAL, M. J. N.; COSTA, K. C. C.; LINS-ESILVA, A. C. B. Estrutura da vegetação caducifólia espinhosa (caatinga) de uma área do sertão central de Pernambuco. Hoehnea, São Paulo, v. 35, p. 209-217, 2008a.

RODAL, M. J. N.; MARTINS, F. R.; SAMPAIO, E. V. S. B. Levantamento quantitativo das plantas lenhosas em trechos de vegetação de caatinga em Pernambuco. Revista Caatinga, v. 21, p. 192-205, 2008b.

SAMPAIO, E. V. S. B. A Vegetação do Bioma Caatinga. In: SAMPAIO, E. V. S. B. et al. (Orgs.). Vegetação e flora da caatinga. Recife: Associação de Plantas do Nordeste e Centro Nordestino de Informações sobre Plantas, 2002. p. 11-24.

SAMPAIO, E. V. S. B. Fitossociologia. In: SAMPAIO, E. V. S. B.; MAYO, S. J.; BARBOSA, E M. R. V. (Eds.). Pesquisa Botânica do Nordeste: progresso e perspectivas. Recife: Sociedade Botânica do Brasil, 1996. 224 p.

SEGURA, G. et al. Tree community structure and stem mortality along a water availability gradient in a Mexican tropical dry forest. Plant Ecology, v. 169, p. 259-271, 2003.

TER BRAAK, C. J. F. Ordination. In: JONGMAN, R. H. G.; TER BRAAK, C. J. F.; VAN TONGEREN, O. F. R. (Eds.). Data analysis in community and landscape ecology. Cambrigde: Cambridge University Press, 1995. p. 91-173.

TREJO, I.; DIRZO, R. Floristic diversity of Mexican seasonally dry tropical forests. Biodiversity Conservation, v. 11, p. 2048-2063, 2002.

VELOSO, H. P. et al. Classificação vegetação brasileira, adaptada a um sistema universal. Rio de Janeiro: IBGE, 1991. 123 p.

WIRTH, R; WEBER, B; RYEL, R. J. Spatial and temporal variability of canopy structure in a tropical moist forest. Acta Oecologia, v. 22, p. 235-244, 2001.

ZAR, J. H. Biostatistical analysis. 4. ed. New Jersey: Prentice-Hall, 1999. 663 p. 